\title{
Importance of High-Frequency Vestibular Function in the Prognosis of Bilateral Vestibulopathy
}

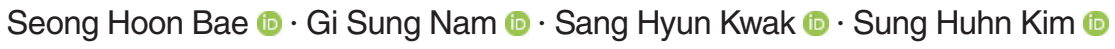 \\ Department of Otorhinolaryngology, Yonsei University College of Medicine, Seoul, Korea
}

Objectives. The aim of this study was to investigate whether preserved vestibular function in the high-frequency range influences the prognosis of patients with bilateral vestibulopathy (BVP) after vestibular rehabilitation.

Methods. Twenty-four patients followed up with vestibular rehabilitation were recruited. The enrolled patients were divided into two groups according to the preservation of the high-frequency vestibulo-ocular reflex (VOR) based on the video head impulse test (vHIT). The results of computerized dynamic posturography and the Dizziness Handicap Inventory (DHI) survey collected at baseline and at the 6-month follow-up after vestibular rehabilitation therapy were analyzed.

Results. Both groups showed significantly increased composite and DHI scores after follow-up with vestibular rehabilitation. The group with preserved high-frequency VOR showed a better composite score $(P=0.064)$ and vestibular score $(P=$ 0.008 ) than the group with lost high-frequency VOR at the 6-month follow up. The DHI score significantly decreased only in the group with lost high-frequency VOR $(P=0.047)$. Among the three vestibular function tests (caloric test, rotary chair test, and vHIT) used to diagnose BVP, only vHIT showed a significant correlation $(P=0.015)$ with a favorable prognosis (composite score $\geq 70$ ).

Conclusion. Better treatment outcomes are likely in patients with BVP with preserved vestibular function in response to high-frequency stimulation, as measured by the vHIT.

Keywords. BilateralVestibulopathy; Head Impulse Test; Vestibular Function Tests; Semicircular Canals; Rehabilitation

\section{INTRODUCTION}

The diagnosis of bilateral vestibulopathy (BVP) was conventionally made based on the complete or near-complete loss of the bilateral vestibulo-ocular reflex (VOR) in the bithermal caloric test and rotary chair test (RCT) [1]; however, the diagnostic criteria varied across institutions, and there were no universally accepted criteria for a definitive diagnosis. Moreover, those two tests mainly assess vestibular function using a low- to mid-frequency angular acceleration stimulation; hence, the preservation of vestibular function in response to high-frequency angular acceleration stimulation can be missed if the diagnosis is made

- Received August 18, 2020

Revised October 12, 2020

Accepted November 13, 2020

- Corresponding author: Sung Huhn Kim

Department of Otorhinolaryngology, Yonsei University College of Medicine,

50-1 Yonsei-ro, Seodaemun-gu, Seoul 03722, Korea

Tel: +82-2-2228-3604, Fax: +82-2-393-0580

E-mail: fledermaus@yuhs.ac solely based upon those two tests [2]. Recently, new diagnostic criteria for BVP were suggested by the Barany Society. These criteria include not only the results of the caloric test and RCT, but also a decrease in gain in the video head impulse test (vHIT), which detects the VOR at high-frequency angular acceleration above $5 \mathrm{~Hz}[3,4]$. The most important aspect of this new paradigm is that it involves an assessment of loss of vestibular function in response to high-frequency stimulation. The inclusion of this test for diagnosing BVP is likely to become mandatory because the range of angular head movement frequencies in daily life is between 1 and $3 \mathrm{~Hz}$.

The prognosis of BVP is poorer than that of unilateral vestibulopathy due to poor vestibular compensation and adaptation as a result of near-complete bilateral vestibular loss $[1,5]$. Therefore, the effect of vestibular rehabilitation on BVP has been thought to be limited. Nevertheless, vestibular rehabilitation therapy is a valuable treatment for patients with BVP because it can enhance sensory substitution such as use of somatosensory and other coping strategies of eye movement for stable vision, such as smooth

Copyright @ 2021 by Korean Society of Otorhinolaryngology-Head and Neck Surgery.

This is an open-access article distributed under the terms of the Creative Commons Attribution Non-Commercial License (https://creativecommons.org/licenses/by-nc/4.0)

which permits unrestricted non-commercial use, distribution, and reproduction in any medium, provided the original work is properly cited. 
Table 1. Diagnostic criteria for bilateral vestibulopathy proposed by the Barany Society

\begin{tabular}{l}
\hline Diagnostic criteria \\
\hline A. Chronic vestibular syndrome with the following symptoms \\
1. Unsteadiness when walking or standing \\
2. Movement-induced blurred vision or oscillopsia during walking or quick head/body movements \\
3. Worsening of unsteadiness in darkness and/or on uneven ground \\
B. No symptoms while sitting or lying down under static conditions \\
C. Bilateral reduced or absent angular VOR function documented by \\
1. Bilaterally pathological horizontal angular VOR gain $<0.6$, measured by the video-head impulse test or scleral-coil technique \\
2. Reduced caloric response (sum of bilateral max; peak slow phase velocity on each side $<6^{\circ} / \mathrm{sec}$ ) \\
3. Reduced horizontal angular VOR gain $<0.1$ upon sinusoidal stimulation on a rotary chair $\left(0.1 \mathrm{~Hz}, \mathrm{Vmax}=50^{\circ} / \mathrm{sec}\right.$ ) and a phase lead $>68^{\circ}$ (time \\
constant $<5$ sec) \\
D. Not better accounted for by another disease
\end{tabular}

VOR, vestibulo-ocular reflex.

pursuit and saccade. Consequently, vestibular rehabilitation can reduce symptoms and prevent falls, and several studies have reported encouraging outcomes [1,5-11].

We hypothesized that there may be differences in the prognosis of BVP patients who receive vestibular rehabilitation depending on whether vestibular function is preserved in high-frequency ranges due to the importance of those ranges for daily life activities. Therefore, we analyzed the results of objective and subjective parameters evaluated by computerized dynamic posturography (CDP) and the Dizziness Handicap Inventory (DHI) between two groups divided according to preserved lateral semicircular canal (LSCC) function in the high-frequency range as determined by vHIT gain in the LSCC. We believe that these results will aid in evaluation and treatment-planning strategies for patients with BVP.

\section{MATERIALS AND METHODS}

The Institutional Review Board of the Severance Hospital approved this study (IRB No. 4-2019-0286). The informed consent was waived because this study design is retrospective medical record analysis.

\section{Patient enrollment}

Twenty-four patients who were newly diagnosed with BVP according to the diagnostic criteria proposed by the Barany Society

\section{H I G G H L I I}

- High frequency preserved bilateral vestibulopathy patients have reduced symptoms.

- High frequency preserved bilateral vestibulopathy patients have a better prognosis.

- Stricter criteria of high frequency preserved bilateral vestibulopathy may be needed.
(Table 1) and who had a minimum follow-up period of more than 6 months were enrolled [12]. The duration of disease was defined as recent if the duration of the symptom was within 6 months and as remote if the duration of the symptom was more than 6 months. There were various causes of BVP: idiopathic $(n=14)$, use of ototoxic drugs $(n=4)$, bilateral Meniere's disease $(n=2)$, bilateral vestibular neuritis $(n=1)$, Cogan syndrome $(n=1), M e-$ niere's disease with contralateral iatrogenic vestibular loss $(n=1)$, and Ramsay Hunt syndrome with contralateral iatrogenic vestibular loss $(n=1)$. Except for hypertension $(\mathrm{HTN}, \mathrm{n}=11)$ and diabetes mellitus (DM, $n=6)$, the patients did not have any other somatic or neurological disorders that could influence the results of the vestibular function tests.

\section{Evaluation of vestibular function}

All patients were diagnosed with BVP after the bithermal caloric test (SLVNG; SLMED, Seoul, Korea), RCT (NOTC-S; Neuro Kinetics, Pittsburgh, PA, USA), and vHIT (ICS Impulse; Otometrics, Taastrup, Denmark) were performed. Those tests were performed by the protocols used in the previous reports [13,14]. In addition to those tests, CDP (SMART EquiTest; Natus Medical Inc, Pleasanton, CA, USA) tests were performed to test balance maintenance. The composite and vestibular scores in the sensory organization test of the CDP were calculated using the weighted average of the equilibrium scores. The cervical and ocular vestibular-evoked myogenic potential (cVEMP and oVEMP) responses were recorded in the ipsilateral sternocleidomastoid muscle (cVEMP) or the contralateral inferior oblique ocular muscle (oVEMP; Audera, GSI, Eden Prairie, MN, USA) by $95 \mathrm{~dB}$ HL, $500 \mathrm{~Hz}$ tone burst stimulation. We categorized the results of the VEMP tests into "presence" and "absence" of the response. All the patients were surveyed using the Korean version of the DHI at initial diagnosis.

\section{Vestibular rehabilitation and follow-up}

None of the enrolled patients had undergone any prior vestibular rehabilitation. The rehabilitation therapy was started within a 
week after the initial diagnosis. They were instructed to perform exercises to improve their oculomotor function, such as saccade and smooth pursuit, by gazing at the horizontally and vertically moving targets at the speed to which they could adjust. Visual adaptation exercises were recommended in addition to the above exercise if patients could tolerate them; the patients were instructed to rotate and move their head up and down while trying to maintain their visual fixation on a target for 1-2 minutes, three times per day. They were also given instructions regarding exercises to improve their posture and gait. The patients were asked to maintain an upright posture with a narrow foot base. If they could tolerate that, they were instructed to remain in an upright position while closing their eyes. After maintaining this posture, they were directed to attempt to march in place. If this exercise could be performed without an issue, the patient was instructed to walk and then to attempt a tandem gait. Horizontal and vertical head movements were then added during the gait exercise. Although the general rehabilitation protocol was as described above, it was modified according to each patient's individual symptoms. After providing the instructions, we sent the patients home with directions to repeat the exercises at least three times per day for 1-2 months. The patients visited an outpatient clinic 1-2 months later, when their exercise diaries were checked, and they were evaluated regarding whether they correctly and consistently performed the exercises by asking the patients to perform the exercises they had learned. Subsequently, the patients were re-educated about the exercises if their performance was insufficient or were given additional instructions if they had adequately performed them. Additional vestibular function tests were selectively conducted depending upon the patients' symptoms and discomfort during the follow-up. The CDP and DHI survey was performed 6 months after the baseline test to identify improvements in balance maintenance and subjective symptoms.

\section{Statistical analysis}

Because the number of patients was small $(n=24)$, nonparametric statistical tests were used.The Mann-Whitney $U$-test was used to compare two independent groups. The Wilcoxon signed-rank test was used to evaluate the outcomes after follow up. Fisher's exact test was used to evaluate proportional significance. The values in the tables and text are presented as the mean \pm standard deviation. A $P$-value $<0.05$ was considered to indicate statistical significance. The statistical analyses were conducted using IBM SPSS ver. 23 (IBM Corp., Armonk, NY, USA).

\section{RESULTS}

\section{Demographic data and vestibular function of the patients}

The patients' mean age was $60.63 \pm 16.26$ years. The mean slow phase velocity (SPV) sum in the bithermal caloric test was $6.56 \pm$
13.95, RCT gain (0.12 Hz slow harmonic acceleration test) was $0.19 \pm 0.16$, and the gain of vHIT in the LSCC (average of both canals) was $0.51 \pm 0.19$. According to the diagnostic criteria of the Barany Society [12], seven of the 24 patients satisfied the criteria of all three tests (caloric test, RCT, and vHIT), six patients satisfied the criteria for two tests (one for the caloric test and RCT, and five for the caloric test and vHIT) and 11 patients satisfied the criteria of only a single test (eight for the caloric test and three for vHIT). The patients were categorized into two groups: group $1(n=9)$ denotes the group with preserved high-frequency VOR, as shown by a gain of VHIT in the LSCC of 0.6 or higher on at least one side, and group $2(n=15)$ denotes the group with loss of high-frequency VOR, as shown by a gain of vHIT in the LSCC of less than 0.6 on both sides. The demographic characteristics and the results of the vestibular function tests of the two groups are listed in Table 2. Age, sex, disease duration, comorbidities of HTN and/or DM, and the results of cVEMP and oVEMP

Table 2. Information on the enrolled patients

\begin{tabular}{|c|c|c|c|c|}
\hline Factor & $\begin{array}{l}\text { Group } 1 \\
(n=9)\end{array}$ & $\begin{array}{l}\text { Group } 2 \\
(n=15)\end{array}$ & $\begin{array}{l}\text { Total } \\
(n=24)\end{array}$ & $P$-value \\
\hline Age (yr) & $58.56 \pm 15.69$ & $61.87 \pm 17.02$ & $60.63 \pm 16.26$ & 0.570 \\
\hline Sex & & & & 0.678 \\
\hline Male & 3 & 7 & 10 & \\
\hline Female & 6 & 8 & 14 & \\
\hline Disease duration ${ }^{\text {a) }}$ & & & & 1.000 \\
\hline Recent & 4 & 7 & 11 & \\
\hline Remote & 5 & 8 & 13 & \\
\hline Hypertension & 4 & 7 & 11 & 1.000 \\
\hline Diabetes mellitus & 1 & 5 & 6 & 0.351 \\
\hline cVEMP loss ${ }^{\mathrm{b})}$ & 5 & 8 & 13 & 1.000 \\
\hline oVEMP loss ${ }^{\text {b) }}$ & 6 & 12 & 18 & 0.635 \\
\hline Caloric SPVc) & $4.17 \pm 6.6$ & $8.0 \pm 17$ & $6.56 \pm 13.95$ & 0.706 \\
\hline RCT SHA gain ${ }^{d)}$ & $0.28 \pm 0.14$ & $0.14 \pm 0.15$ & $0.19 \pm 0.16$ & $0.022^{*}$ \\
\hline $\begin{array}{l}\text { Average vHIT } \\
\text { gaine) }^{\mathrm{e}}\end{array}$ & $0.70 \pm 0.10$ & $0.39 \pm 0.12$ & $0.51 \pm 0.19$ & $<0.001^{*}$ \\
\hline
\end{tabular}

Values are presented as mean \pm standard deviation. Group 1 and group 2 included patients with preservation or loss of high-frequency VOR (LSCC gain $\geq 0.6$ at least on one side or LSCC gain $<0.6$ on both sides on the $\mathrm{VHIT})$, respectively.

cVEMP, cervical vestibular-evoked myogenic potential; oVEMP, ocular vestibular-evoked myogenic potential; SPV, slow-phase velocity; RCT, rotary chair test; SHA, slow harmonic acceleration; VHIT, video head impulse test; VOR, vestibulo-ocular reflex; LSCC, lateral semicircular canal.

a)Duration of disease was defined as recent if the duration of the symptoms was within 6 months and as remote if the duration of the symptoms was more than 6 months. ${ }^{\text {b) }}$ VVEMP/oVEMP loss indicated bilateral "no response" in cervical or ocular vestibular-evoked myogenic potential. " $\mathrm{Ca}$ loric SPV indicated the sum of the SPV of nystagmus evoked by bilateral warm and cold-water stimulation. ${ }^{\text {d) }} \mathrm{RCT}$ SHA gain indicated the gain at $0.12-\mathrm{Hz}$ slow harmonic acceleration stimulation in the RCT. ${ }^{\mathrm{e}} \mathrm{VHIT}$ gain indicated the average gain of the bilateral LSCC.

The significance of differences in sex, duration, hypertension, diabetes mellitus, and cVEMP/oVEMP loss between the two groups was analyzed using the Fisher exact test. Other factors were analyzed using the MannWhitney U-test. ${ }^{*} P<0.05$. 

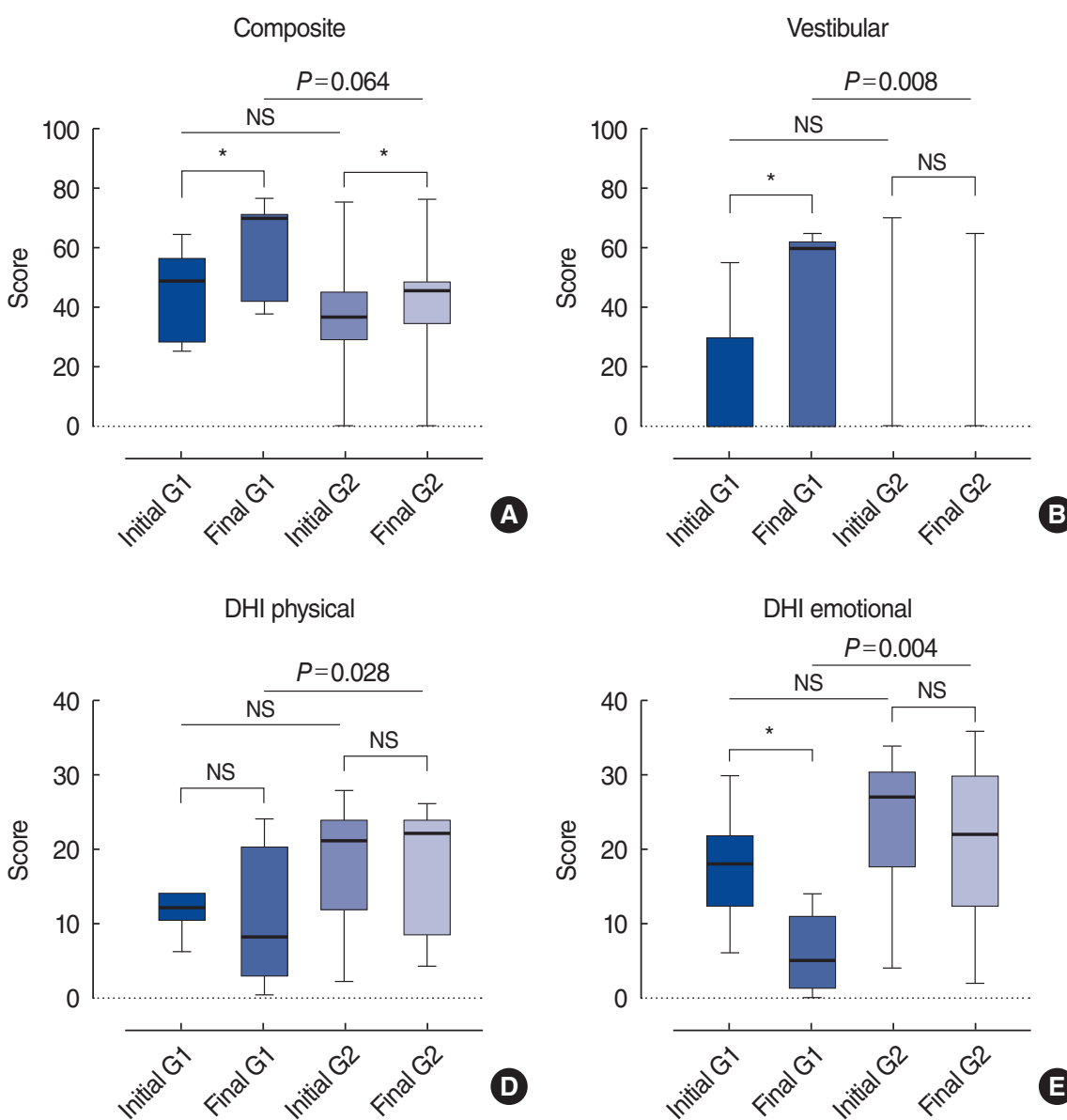

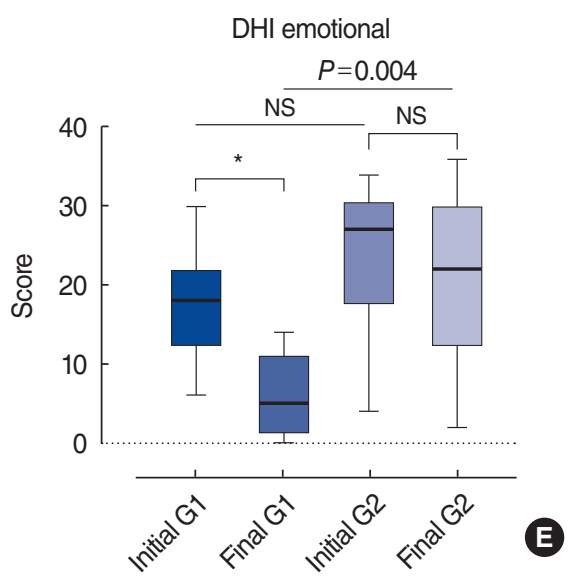

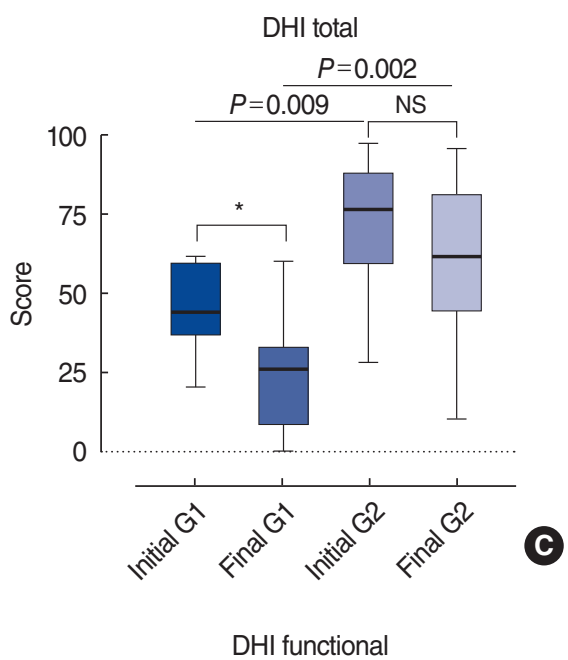

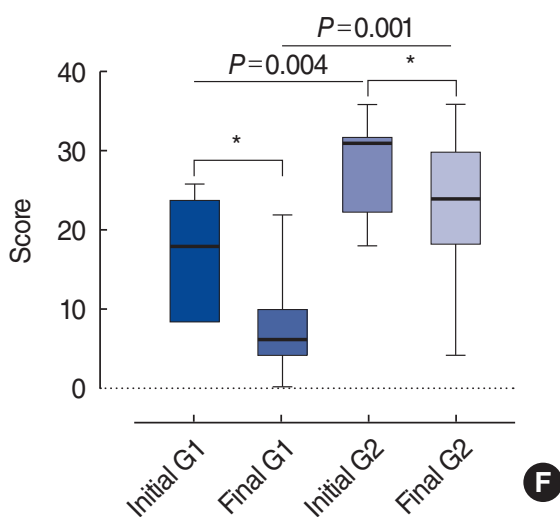

Fig. 1. Changes in subjective symptoms and objective parameters of balance function after rehabilitation. A simple boxplot of the computerized dynamic posturography and Dizziness Handicap Inventory (DHI) scores for bilateral vestibulopathy patients at baseline and follow-up. G1 (group 1, video head impulse test [VHIT] gain in the lateral semicircular canal [LSCC] $\geq 0.6$ in one or both ears) patients showed a better outcome than G2 (group 2, vHIT gain in the LSCC <0.6 in both ears) patients. (A) Composite score, (B) vestibular score, (C) DHI total score, (D) DHI physical score, (E) DHI emotional score, and (F) DHI functional score. The line in the middle is the median. The top and bottom box lines show the first and third quartiles, respectively. The whiskers show the maximum and minimum values. NS, not significant. ${ }^{*} P<0.05$.

were not significantly different between the two groups $(P>0.05)$. The sum of SPV in the bithermal caloric test of both ears was not significantly different $(P=0.706)$; however, the gain of RCT showed significant differences between the two groups $(P=0.022)$. There were no significant between-group differences in the initial composite score or vestibular score $(P>0.05)$ (Fig. 1). However, the initial subjective parameters, as denoted by the total and functional DHI scores, showed significant differences between the two groups ( $P=0.007$ and $P=0.003$, respectively) (Fig. 1). This finding implies that the preservation of high-frequency vestibular function can diminish patients' subjective symptoms.

\section{Prognostic differences according to high-frequency VOR preservation}

Objective and subjective improvements of balance function were measured by comparing the changes in the vestibular score and composite score of CDP, and by comparing changes in DHI scores in the patients in each group after 6 months of follow-up (Table
3). After rehabilitation, the composite score of CDP significantly improved in both groups $(P=0.016$ and $P=0.023$ for groups 1 and 2 , respectively). The score improvement was somewhat higher in group 1 than in group 2, but the difference between the two groups was not statistically significant $(P=0.064)$. However, the vestibular score significantly improved after rehabilitation only in group $1(P=0.031)$, and the score improvement was significantly higher in group 1 than in group $2(P=0.002)$. The total DHI score significantly improved after rehabilitation in group 1 $(P=0.047)$, but not in group 2 . Among the subcategories of the DHI survey, the physical DHI score showed no significant improvement in either group, the emotional DHI score significantly improved only in group $1(P=0.016)$, and the functional DHI score significantly improved in both groups (group $1, P=0.031$; group $2, P=0.005)$. The total and all the subcategories of the final DHI scores were significantly lower in group 1 than in group 2 at follow up $(P=0.001, P=0.026, P=0.002$, and $P<0.001$ for total, physical, emotional, and functional DHI scores, respective- 
Table 3. Changes in CDP and DHI scores from baseline to follow-up

\begin{tabular}{|c|c|c|c|c|}
\hline Variable & Group 1 & Group 2 & Total & $P$-value \\
\hline \multicolumn{5}{|l|}{ PTV } \\
\hline Change & $28.3 \pm 28.4$ & $-2.3 \pm 10.8$ & $9.2 \pm 24.1$ & $0.002^{*}$ \\
\hline$P$-value ${ }^{\text {a) }}$ & $0.031^{*}$ & 0.750 & 0.066 & \\
\hline \multicolumn{5}{|l|}{ PTc } \\
\hline Change & $16.3 \pm 14.8$ & $5.6 \pm 9.1$ & $9.6 \pm 12.4$ & 0.064 \\
\hline$P$-value ${ }^{\text {a) }}$ & $0.016^{*}$ & $0.023^{*}$ & $0.001^{*}$ & \\
\hline \multicolumn{5}{|l|}{ DHI total } \\
\hline Change & $-26.3 \pm 23.8$ & $-10.4 \pm 17.4$ & $-15.7 \pm 20.6$ & 0.125 \\
\hline$P$-value ${ }^{\text {a) }}$ & $0.047^{\star}$ & 0.080 & $0.006^{\star}$ & \\
\hline \multicolumn{5}{|c|}{ DHI physical } \\
\hline Change & $-2.9 \pm 9.6$ & $0.1 \pm 5.8$ & $-0.9 \pm 7.2$ & 0.202 \\
\hline$P$-value ${ }^{\text {a) }}$ & 0.406 & 0.970 & 0.467 & \\
\hline \multicolumn{5}{|c|}{ DHI emotional } \\
\hline Change & $-12.1 \pm 9.2$ & $-3.4 \pm 8.9$ & $-6.3 \pm 9.7$ & 0.052 \\
\hline$P$-value ${ }^{\text {a) }}$ & $0.016^{\star}$ & 0.235 & $0.009^{*}$ & \\
\hline \multicolumn{5}{|c|}{ DHI functional } \\
\hline Change & $-11.3 \pm 8.9$ & $-5.7 \pm 6.9$ & $-7.6 \pm 7.9$ & 0.133 \\
\hline$P$-value ${ }^{\text {a) }}$ & $0.031^{*}$ & $0.005^{*}$ & $<0.001^{*}$ & \\
\hline
\end{tabular}

Values are presented as mean \pm standard deviation. Group 1 and group 2 included patients with preservation or loss of high-frequency VOR (LSCC gain $\geq 0.6$ at least on one side or LSCC gain $<0.6$ on both sides on the $\mathrm{VHIT})$, respectively.

CDP, computerized dynamic posturography; DHI, Dizziness Handicap Inventory; PTv, vestibular score in posturography; PTc, composite score in posturography; VOR, vestibulo-ocular reflex; LSCC, lateral semicircular canal.

The Mann-Whitney U-test was used to evaluate differences between group 1 and group 2. The Wilcoxon signed-rank test was used to evaluate differences between baseline and follow-up. a) $P$-value calculated by the Wilcoxon signed-rank test to evaluate the statistical significance of differences between initial and final values. ${ }^{*} P<0.05$.

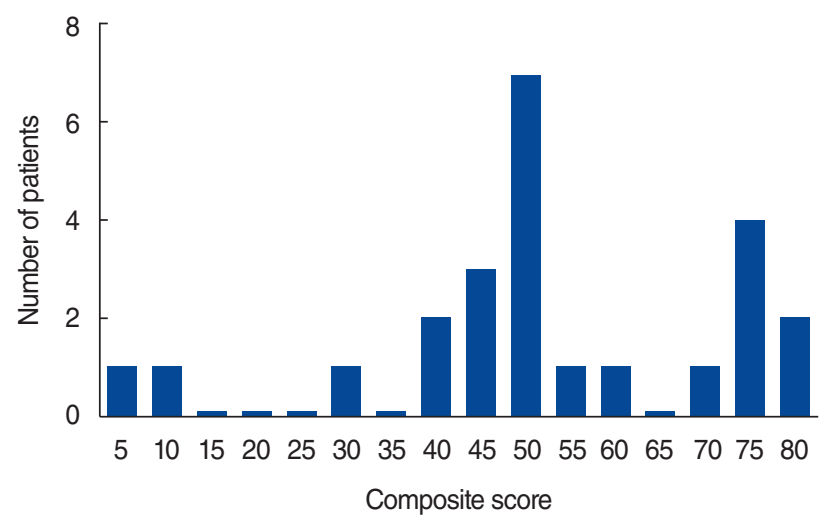

Fig. 2. Distribution of patients according to the final composite score after vestibular rehabilitation. The cumulative graph of the final composite score showed a double peak at 45-50 and 70-75.

ly) (Fig. 1C-F), however, the score improvement was not significant in any DHI subcategory.

Altogether, BVP patients showed improved postural function and subjective symptoms after 6 months of follow-up with vestibular rehabilitation, and the patients with preserved high-fre-
Table 4. Comparison of patient groups divided according to prognosis

\begin{tabular}{|c|c|c|c|}
\hline Variable & $\begin{array}{l}\text { Favorable } \\
\qquad(n=6)\end{array}$ & $\begin{array}{l}\text { Unfavorable } \\
\qquad(n=18)\end{array}$ & $P$-value \\
\hline Age (yr) & $62.83 \pm 10.17$ & $59.89 \pm 18.03$ & 0.871 \\
\hline Sex & & & 0.341 \\
\hline Male & 1 & 9 & \\
\hline Female & 5 & 9 & \\
\hline Duration of disease ${ }^{a)}$ & & & 1.000 \\
\hline Recent & 3 & 8 & \\
\hline Remote & 3 & 10 & \\
\hline Hypertension & & & 0.649 \\
\hline Yes & 2 & 9 & \\
\hline No & 4 & 9 & \\
\hline Diabetes mellitus & & & 1.000 \\
\hline Yes & 1 & 5 & \\
\hline No & 5 & 13 & \\
\hline cVEMP & & & 1.000 \\
\hline Loss $^{\text {b) }}$ & 3 & 10 & \\
\hline Present & 3 & 8 & \\
\hline oVEMP & & & 0.139 \\
\hline Loss $^{b)}$ & 3 & 15 & \\
\hline Present & 3 & 3 & \\
\hline Caloric test ${ }^{c)}$ & & & 1.000 \\
\hline Loss & 5 & 16 & \\
\hline Present & 1 & 2 & \\
\hline $\mathrm{RCT}^{\mathrm{d})}$ & & & 0.066 \\
\hline Loss & 0 & 8 & \\
\hline Present & 6 & 10 & \\
\hline $\mathrm{VH} I \mathrm{~T}^{\mathrm{e})}$ & & & $0.015^{\star}$ \\
\hline Loss & 1 & 14 & \\
\hline Present & 5 & 4 & \\
\hline
\end{tabular}

The favorable group and unfavorable group included patients with a composite score of 70 or more and less than 70 , respectively, at 6 months of follow-up.

cVEMP, cervical vestibular-evoked myogenic potential; oVEMP, ocular vestibular-evoked myogenic potential; RCT, rotary chair test; vHIT, video head impulse test.

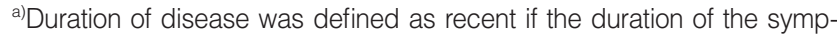
toms was within 6 months and as remote if the duration of the symptoms was more than 6 months. ${ }^{b}$ CVEMP/oVEMP loss indicated bilateral "no response" in cervical and ocular vestibular-evoked myogenic potentials. cLoss in the caloric test indicated that the sum of the slow phase velocity of nystagmus on each side evoked by cold and warm water stimulation was lower than $6 \% \mathrm{sec}$. "Present" in the caloric test indicated that the sum of the slow-phase velocity of nystagmus evoked by cold and warm water stimulation was $6 \% \mathrm{sec}$ or more at least in one ear. ${ }^{d)}$ "Loss" in the RCT indicated that the gain at $0.12 \mathrm{~Hz}$ of slow harmonic acceleration stimulation was lower than 0.1 in the RCT. "Present" in the RCT indicated that the gain at $0.12 \mathrm{~Hz}$ of slow harmonic acceleration stimulation was 0.1 or more. e)"Loss" in the VHIT indicates that the gain of the lateral semicircular canal was lower than 0.6 in both sides of the ear in the VHIT. "Present" in the VHIT indicated that the gain of the lateral semicircular canal was 0.6 or higher in one or both sides of the ear in the VHIT.

The significance of the difference in age between the two groups was analyzed using the Mann-Whitney U-test. Other factors were analyzed using the Fisher exact test. ${ }^{*} P<0.05$ 
quency VOR tended to show more favorable outcomes, with significantly improved vestibular scores.

\section{Characteristics of patients who showed a favorable prognosis}

To confirm the importance of high-frequency VOR in the prognosis of BVP, we investigated which of the initial vestibular function tests could predict the prognosis. Since the final composite scores of BVP patients of this study showed two peaks, at 45-50 and 70-75 (Fig. 2), a composite score of 70 or higher was considered to indicate a favorable prognosis $[15,16]$. We categorized the patients with favorable and unfavorable prognoses into contingency tables with the other parameters of demographic factors, systemic disease condition, and the results of vestibular function tests (Table 4). Age, sex, disease duration, and presence of HTN and/or DM were not significantly different between the two groups $(P>0.05)$. In the vestibular function tests, only a preserved VOR gain equal to or higher than 0.6 in the vHIT showed a significant correlation with a favorable prognosis $(P=0.015)$.

\section{DISCUSSION}

Previous studies have suggested that vestibular rehabilitation therapy was effective, but had limited effects on the improvement of balance function in patients with BVP [1,5]. The results of this study are in accordance with the results of those prior studies. Although the average composite score tended to increase after follow-up with vestibular rehabilitation, only seven of the 24 patients $(29 \%)$ showed recovery of a CDP composite score of 65 or more. The most salient difference between the patients with a composite score equal to or greater than 65 and the others was revealed to be the preservation of vestibular function in the highfrequency stimulation range, which could be identified by the vHIT. In other words, patients with preserved high-frequency VOR showed better outcomes after rehabilitation.

It is well known that the efficiency of the VOR against lowfrequency stimulation is poorer than that against the higher-frequency range $[17,18]$, as proven by a mechanical model and physical interpretation $[19,20]$. Since the results from the vHIT represent the VOR at a high-frequency stimulation range $(5-7 \mathrm{~Hz})$ that is closer to the VOR that occurs in daily life $[21,22]$, it is reasonable to infer that the preservation of high-frequency VOR in BVP patients is important both for decreasing the severity of subjective symptoms and for promoting the recovery of balance function in patients with BVP after vestibular rehabilitation. However, the caloric test, which presents non-physiological low-frequency stimulation $(<0.005 \mathrm{~Hz})$, is likely to have less importance than the vHIT in anticipating patients' prognosis after vestibular rehabilitation. These assumptions are supported by the results of this study. Initially, the group with lost high-frequency VOR (group 2) showed more severe subjective symptoms according to the initial DHI score and did not exhibit any significant differences in the initial composite score and vestibular score in CDP, unlike the patients with preserved high-frequency VOR (group 1). In addition, the patients with preserved high-frequency VOR (group 1) showed a certain amount of preserved VOR gain in the RCT compared to the patients with lost high-frequency VOR (group 2). This result implies that subjective symptoms could be more severe in patients who lose high-frequency VOR than in those with preserved high-frequency VOR. Even though the patients had a preserved VOR in the mid- to high-frequency range, their static and dynamic balance function, as measured using CDP, was very poor due to their bilateral vestibular loss, which resulted in a significant physical disability compared to that associated with unilateral vestibular function loss. Hence, the scores were not different between group 1 and group 2 at baseline. After rehabilitation, patients with preserved high-frequency VOR showed more significant improvement in the vestibular score and tended to have favorable final outcomes in other parameters. If bilateral vestibular function is completely lost, vestibular compensation cannot be achieved; instead, improvements in balance function depend on substitution using other sensory information such as vision and somatosensory cues $[23,24]$. However, these substitution strategies do not provide enough balance function maintenance during high-frequency head and body movements because the frequency range covered by sensory substitution is lower than that of vestibular reflexes. Therefore, patients with partially preserved vestibular function in response to mid- to high-frequency ranges of stimulation can accomplish vestibular compensation more efficiently. The observation in this study that patients who showed a caloric test response above the threshold of the diagnostic criteria for BPV, but without a vHIT response, showed a poorer final composite score than patients without a caloric response, but with a preserved vHIT response, also supports this hypothesis. Considering the results of VOR preservation in the RCT in group 1, the results of the RCT seemed to be another contributing factor to the prognosis. Therefore, we categorized the patients into a final high composite score group ("favorable group") and low composite score group ("unfavorable group") and tried to identify differences in the various factors. In the comparison, the proportion of patients with preserved high-frequency VOR was significantly larger in the favorable group than in the unfavorable group. Although the proportion of patients with preserved gain in the RCT was higher in the favorable group than in the unfavorable group, the difference was not statistically significant. This may have resulted from the relatively small number of enrolled patients. The incidence of BVP is very low $(28$ per 100,000$)$, and it was difficult to recruit a large number of patients at a single institution to reveal the specific relationship between the results of RCT and prognosis [12].

For vestibular rehabilitation in BPV patients, a physician and/ or physical therapist can strongly recommend adaptation exercises for vision stabilization combined with substitution exercises for BVP patients with preserved high-frequency VOR gain. In 
patients with total BVP, the effect of adaptation exercises is expected to be minimal; therefore, vision stabilization exercises reinforcing saccadic or smooth-pursuit oculomotor function should be used instead of theVOR. We recommended an adaptation exercise to all the enrolled patients, but it is likely that the exercise was effective in limited cases. Nonetheless, the exercise seemed to be effective in patients with preserved high-frequency VOR, as can be seen from the results of this study. Therefore, an adaptation exercise may be essential for the rehabilitation of BVP patients with preserved high-frequency VOR gain.

The main limitation of this study is the small number of enrolled patients due to the low incidence of BVP, as described above. Even though we found that the vHIT of the LSCC was significantly correlated with the prognosis, it remains possible that other related variables were not identified due to the lack of statistical power. In addition, we did not perform caloric tests during follow-up due to the expense and patients' discomfort associated with regular administration of caloric tests. Therefore, we could not fully exclude the possibility of recovery of vestibular function during the follow-up period, which may be a cause of a good prognosis.

In conclusion, we found that preservation of the high-frequency range in vestibular function seems to be important for achieving a good prognosis, and the vHIT may be a critical test by which to anticipate the prognosis of BVP. Based on the results of the present study, the possible need for stricter diagnostic criteria of BVP may be discussed in future research.

\section{CONFLICT OF INTEREST}

No potential conflict of interest relevant to this article was reported.

\section{ACKNOWLEDGMENTS}

This study was supported by a faculty research grant of Yonsei University College of Medicine for 6-2020-0153 (granted to SHB) and the Basic Science Research Program through the National Research Foundation of Korea (NRF) funded by the Ministry of Education (NRF-2020R1I1A1A01067241, granted to SHB).

\section{ORCID}

Seong Hoon Bae Gi Sung Nam Sang Hyun Kwak Sung Huhn Kim https://orcid.org/0000-0001-9243-9392 https://orcid.org/0000-0002-8392-5759 https://orcid.org/0000-0002-2827-8456 https://orcid.org/0000-0003-4408-3066

\section{AUTHOR CONTRIBUTIONS}

Conceptualization: SHB, SHK (Sung Huhn Kim). Data curation: SHK (Sang Hyun Kwak), GSN. Formal analysis: SHB. Funding acquisition: SHB. Methodology: SHB, GSN. Project administration: SHB. Visualization: SHB, SHK (Sang Hyun Kwak). Writing-original draft: SHB, SHK (Sung Huhn Kim). Writing-review \& editing: SHB, SHK (Sung Huhn Kim).

\section{REFERENCES}

1. Zingler VC, Weintz E, Jahn K, Mike A, Huppert D, Rettinger N, et al. Follow-up of vestibular function in bilateral vestibulopathy. J Neurol Neurosurg Psychiatry. 2008 Mar;79(3):284-8.

2. Minor LB, Goldberg JM. Influence of static head position on the horizontal nystagmus evoked by caloric, rotational and optokinetic stimulation in the squirrel monkey. Exp Brain Res. 1990;82(1):1-13.

3. Alhabib SF, Saliba I.Video head impulse test: a review of the literature. Eur Arch Otorhinolaryngol. 2017 Mar;274(3):1215-22.

4. Halmagyi GM, Chen L, MacDougall HG, Weber KP, McGarvie LA, Curthoys IS. The video head impulse test. Front Neurol. 2017 Jun 9;8:258.

5. Gillespie MB, Minor LB. Prognosis in bilateral vestibular hypofunction. Laryngoscope. 1999 Jan;109(1):35-41.

6. Lacour M, Bernard-Demanze L. Interaction between vestibular compensation mechanisms and vestibular rehabilitation therapy: 10 recommendations for optimal functional recovery. Front Neurol. 2015 Jan;5:285.

7. Minor LB. Gentamicin-induced bilateral vestibular hypofunction. JAMA. 1998 Feb;279(7):541-4.

8. Krebs DE, Gill-Body KM, Riley PO, Parker SW. Double-blind, placebo-controlled trial of rehabilitation for bilateral vestibular hypofunction: preliminary report. Otolaryngol Head Neck Surg. 1993 Oct; 109(4):735-41.

9. Porciuncula F, Johnson CC, Glickman LB. The effect of vestibular rehabilitation on adults with bilateral vestibular hypofunction: a systematic review. JVestib Res. 2012;22(5-6):283-98.

10. Schubert MC, MigliaccioAA, Clendaniel RA,AllakA, Carey JP. Mechanism of dynamic visual acuity recovery with vestibular rehabilitation. Arch Phys Med Rehabil. 2008 Mar;89(3):500-7.

11. Herssens N,Verbecque E, McCrum C, Meijer K, van de Berg R, Saeys $\mathrm{W}$, et al. A systematic review on balance performance in patients with bilateral vestibulopathy. Phys Ther. 2020 Aug;100(9):1582-94.

12. Strupp M, Kim JS, MurofushiT, Straumann D, Jen JC, Rosengren SM, et al. Bilateral vestibulopathy: diagnostic criteria consensus document of the classification Committee of the Barany Society. J Vestib Res. 2017;27(4):177-89.

13. Jeong J, Jung J, Lee JM, Suh MJ, Kwak SH, Kim SH. Effects of saccular function on recovery of subjective dizziness after vestibular rehabilitation. Otol Neurotol. 2017 Aug;38(7):1017-23.

14. Jung J, Suh MJ, Kim SH. Discrepancies between video head impulse and caloric tests in patients with enlarged vestibular aqueduct. Laryngoscope. 2017 Apr;127(4):921-6.

15. Oda DT, Gananca CF. Computerized dynamic posturography in the assessment of body balance in individuals with vestibular dysfunction. Audiol Commun Res. 2015;20(2):89-95.

16. Gera G, Freeman DL, Blackinton MT, Horak FB, King L. Identification of balance deficits in people with Parkinson disease: is the sensory organization test enough? Int J Phys Med Rehabil. 2016 Feb; 4(1):322. 
17. Demer JL, Oas JG, Baloh RW.Visual-vestibular interaction in humans during active and passive, vertical head movement. JVestib Res. 1993 Summer;3(2):101-14.

18. Meyer CH, LaskerAG, Robinson DA.The upper limit of human smooth pursuit velocity. Vision Res. 1985;25(4):561-3.

19. Rey-Martinez J, Batuecas-Caletrio A, Matino E, Trinidad-Ruiz G, Altuna X, Perez-Fernandez N. Mathematical methods for measuring the visually enhanced vestibulo-ocular reflex and preliminary results from healthy subjects and patient groups. Front Neurol. 2018 Feb;9:69.

20. Roy FD, Tomlinson RD. Characterization of the vestibulo-ocular reflex evoked by high-velocity movements. Laryngoscope. $2004 \mathrm{Jul}$; 114(7):1190-3.
21. Grossman GE, Leigh RJ,Abel LA, Lanska DJ,Thurston SE. Frequency and velocity of rotational head perturbations during locomotion. Exp Brain Res. 1988;70(3):470-6.

22. Hirasaki E, Moore ST, Raphan T, Cohen B. Effects of walking velocity on vertical head and body movements during locomotion. Exp Brain Res. 1999 Jul;127(2):117-30.

23. Herdman SJ, Schubert MC, Tusa RJ. Role of central preprogramming in dynamic visual acuity with vestibular loss. Arch Otolaryngol Head Neck Surg. 2001 Oct;127(10):1205-10.

24. Heimbrand S, Bronstein AM, Gresty MA, Faldon ME. Optically induced plasticity of the cervico-ocular reflex in patients with bilateral absence of vestibular function. Exp Brain Res. 1996 Dec;112(3): 372-80. 